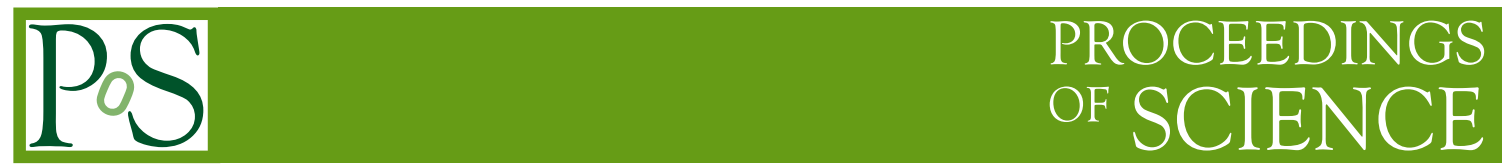

\title{
Correlation of IceCube neutrinos with 2MRS
}

\section{Stephen Sclafani, for the IceCube Collaboration*}

Drexel University, 3141 Chestnut Street, Philadelphia, PA 19104, USA

E-mail: ssclafaniaicecube.wisc.edu

The distribution of galaxies within the local $(\mathrm{z}<0.1)$ universe is characterized by large scale anisotropic features. Observatories searching for the production of astrophysical neutrinos can take advantage of these to establish possible directional correlation between astrophysical neutrinos and galaxy distribution. Here, we present the results of an analysis of the correlation between IceCube neutrinos and galaxy density, as observed by the 2MASS Redshift Survey (2MRS), testing the hypothesis that neutrinos are produced in interactions between diffuse Ultra-High Energy Cosmic Rays (UHECR) and the matter distribution within galaxies. No such correlation was found and limits are placed on neutrino production from diffuse UHECR interacting with local matter.

The New Era of Multi-Messenger Astrophysics - Asterics2019

25 - 29 March, 2019

Groningen, The Netherlands

${ }^{*}$ Speaker. 


\section{Introduction}

IceCube, a cubic kilometer detector at the geographic south pole, has discovered a flux of astrophysical neutrinos [1,2], and more recently, presented compelling evidence for one neutrino source [3,4]. Previous searches, however, have not been able to identify a source population responsible for the majority of the astrophysical neutrino flux. Existing IceCube searches have focused on emission from distant but bright source such as blazars [9] or close large scale features like the galactic plane [7]. This work searches for neutrinos produced in interactions with a diffuse and mostly isotropic flux of UHECR produced elsewhere. If this diffuse UHECR flux were responsible for significant neutrino flux through interactions in target galaxies, the neutrino production would follow the local matter distribution and therefore directional correlation would be observable between the overdensities of the local universe and excesses of astrophysical neutrinos. This work searches for that correlation, analyzing IceCube neutrino data with an extensive local galaxy survey searching for correlation that would be the signifier of these interactions.

\section{2. $2 M R S$}

The 2MASS redshift survey (2MRS) is an all-sky catalog of local galaxies with associated redshift measurements, and is the most extensive and unbiased survey up to redshift of $z<0.03$ [5]. The infrared survey contains the position, redshift and $K_{s}$ band magnitude of 44,000 of the nearest galaxies including galaxies out to a redshift of $z \approx 0.10$. It is mostly complete to a redshift of $z=0.03$ and magnitude limit becomes dominant for further galaxies. The catalog density as a function of direction and redshift is shown in Figure 1. The 2MRS galaxy catalog can be used as a tracer of the distribution of matter in the local universe.
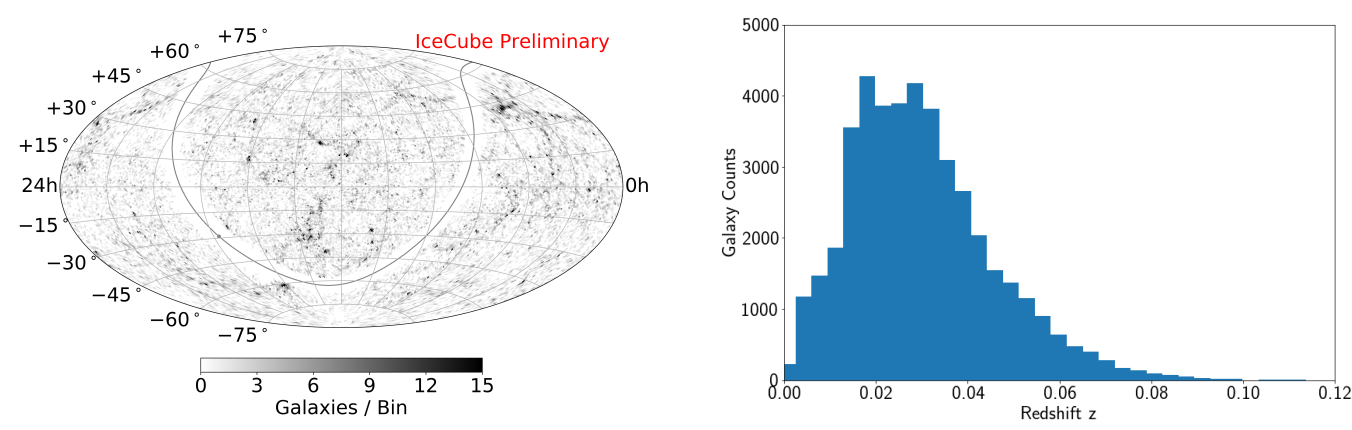

Figure 1: a) Galaxy density of the complete 2MRS Survey and b) Histogram of galaxies measured redshift, showing the completeness and ultimate magnitude limit as a function of redshift

\section{Analysis}

The goal of this analysis is to find statistical correlations between directions of astrophysical neutrinos and 2MRS local galaxy density. A neutrino dataset consisting of 7 years of IceCube muon neutrino events is used that has been previously used in IceCube searches [6]. A Test Statistic (TS) is defined, describing the similarity between the spatial distribution of neutrinos to the 
spatial template of galaxy densities obtained from 2MRS. The energies of the neutrino events are also considered in the TS, and neutrinos with higher energies are more likely to be astrophysical and weighted higher. The background events in IceCube, atmospheric neutrinos and muons, are isotropically distributed within a declination band due to the detector being at the geographic South Pole, thus the statistical significance of the correlation found in the data is quantified by comparing the TS to other TS obtained from data sets events uniformly distributed in right ascension.

This analysis is based on a likelihood function [8] given by a product over all events in the dataset. Detailed descriptions of the method are described in [7], while the construction of the hypothesis specific PDFs are described in section 4. The likelihood, $L$, is a function of $n_{s}$ the number of signal neutrinos, which is free to vary between 0 and the total number of neutrinos. The entire dataset is fit for the most likely $n_{s}$. The test statistic is defined as:

$$
\mathrm{TS}=-2 \ln \left(\frac{L\left(n_{s}=0\right)}{L\left(n_{s}\right)}\right)
$$

where $L\left(n_{s}=0\right)$ is the likelihood for the hypothesis corresponding to no correlation, and $L\left(n_{s}\right)$ is the likelihood for the best fit $n_{s}$. TS is then converted to a $p$-value using simulated isotropic datasets.

\section{Signal PDF Construction}

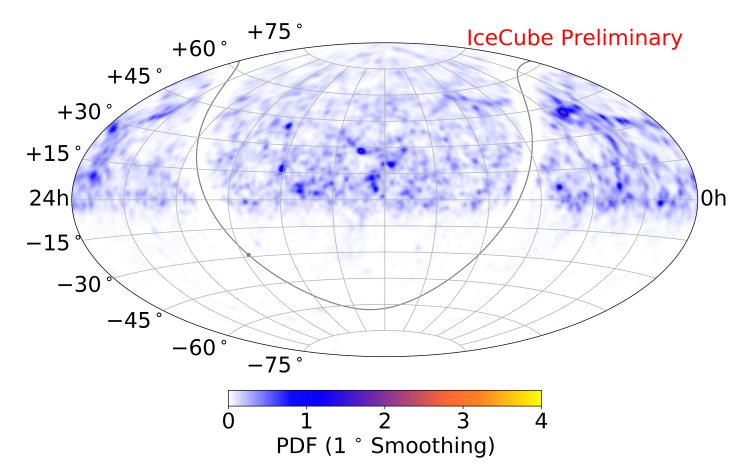

a) Full 2MRS Catalog

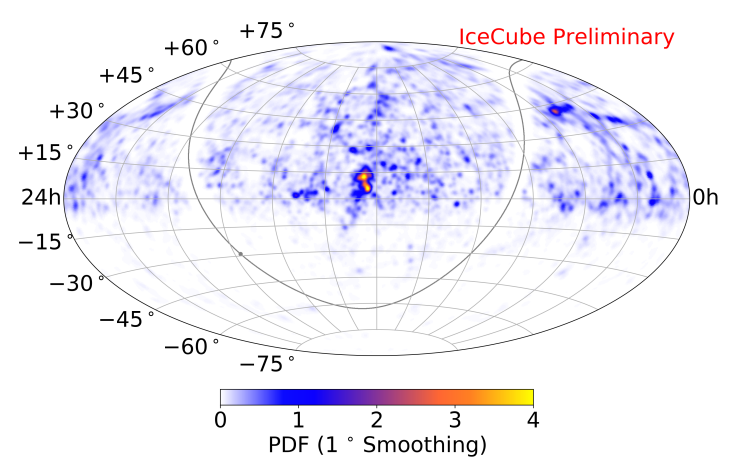

b) Full 2MRS Catalog, weighted by redshift

Figure 2: Signal spatial PDF for a) Full 2MRS Catalog, and b) Full 2MRS Catalog weighted by redshift. Galaxies located in the center of the map make up the Virgo Supercluster and are given extra weight when redshift information is included.

The templates are based on selection and cuts which test different hypotheses. These are shown in Figure 2 and correspond to:

- Full 2MRS Catalog; all equally weighted

- 2MRS Catalog with galaxies of $z<0.03$; all equally weighted

- Full 2MRS Catalog; weighted by redshift distance 
The weighting schemes are chosen to probe specific correlation scenarios. The template with a cutoff at $z=0.03$ corresponds to a close but relatively complete catalog. The redshift-weighted catalog includes weighting proportional to the inverse square of distance where distance is calculated from the redshift, however this does not include any corrections for galaxy peculiar velocity effects or redshift uncertainty. The full catalog uses the complete survey with equal weighting.

Galaxies are binned using equal-area bins of 0.2 square degrees. The density maps are then weighted by each of three hypotheses. The PDF is based on the galaxy density map, convolved with the detector acceptance for an $E^{-2}$ hypothesis as a function of $\sin (\delta)$. The resulting map is then convolved with a Gaussian of width equal to each event angular uncertainty.

\section{Results}

The p-values reported below denote the confidence with which the no-correlation hypothesis can be rejected, and are calculated by integrating over the background trial survival function. All three correlation scenarios indicate an underfluctuation, and are entirely consistent with the no-correlation hypothesis. Given the absence of a significant correlation, limits are placed on the neutrino flux that can originate from local galaxy emission under these three hypothesis. These limits are reported below for each template weighting based on the analysis spectral assumption and shape.

\begin{tabular}{|c|c|c|c|}
\hline Template & Test Statistic & $\mathrm{p}$-value & $\begin{array}{c}\text { Upper Limit } \Phi_{90 \%} \\
\mathrm{dN} / \mathrm{dE}\left(\mathrm{GeV}^{-1} \mathrm{~s}^{-1} \mathrm{~cm}^{-2}\right)\end{array}$ \\
\hline Full Catalog Template & 0.0 & 1.0 & $2.93 \times 10^{-18}$ \\
\hline$z<0.03$ Template & 0.0 & 1.0 & $2.15 \times 10^{-18}$ \\
\hline $\begin{array}{c}\text { Full Catalog Template } \\
\text { with redshift weighting }\end{array}$ & 0.0 & 1.0 & $1.95 \times 10^{-18}$ \\
\hline
\end{tabular}

Table 1: Analysis TS and p-value results. Fluxes are integrated over the full sky and parameterized as $\mathrm{dN} / \mathrm{dE}=\Phi_{90 \%} \times\left(\frac{E}{100 \mathrm{TeV}}\right)^{-2} \mathrm{GeV}^{-1} \mathrm{~cm}^{-2} \mathrm{~s}^{-1}$ with $90 \%$ confidence level upper limits assuming an $\mathrm{E}^{-2}$ spectrum.

Since these limits correspond to very specific hypotheses, we also scanned the test statistic response to other spectral index hypotheses. A likelihood scan showing the change in test statistic as a function of flux at $100 \mathrm{TeV}$ and spectral index is shown in Figure 3 for the full catalog template. Other templates produced similar limits.

This analysis looked for correlation with the 2MRS local universe to search for neutrino production through interactions with a diffuse neutrino flux, and found no correlation. The sensitivity of this analysis will continue to improve as additional surveys provide a more complete view of the local universe. 


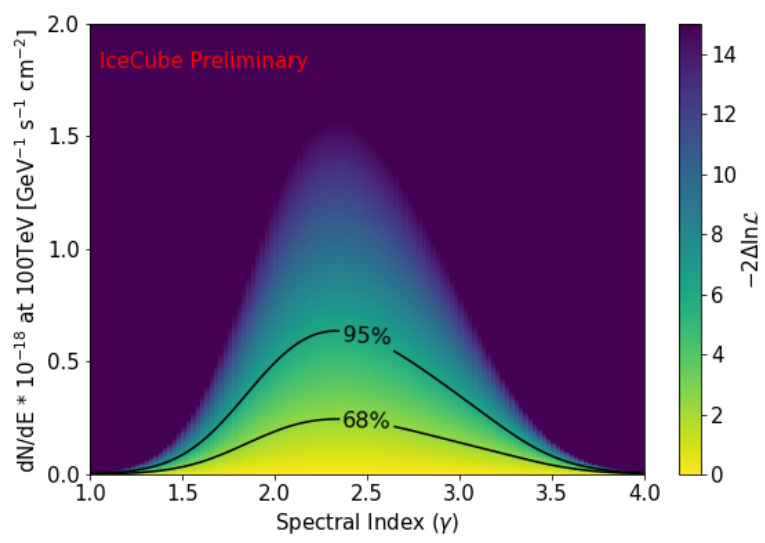

Figure 3: Change in likelihood as a function of spectral index $\gamma$ and flux at $100 \mathrm{TeV}$. Upper limits on neutrino flux a placed at $1 \sigma$ and $95 \%$ confidence intervals

\section{References}

[1] M. G. Aartsen et al. [IceCube Collaboration], Phys. Rev. Lett. 113, 101101 (2014) doi:10.1103/PhysRevLett.113.101101 [arXiv:1405.5303 [astro-ph.HE]].

[2] M. G. Aartsen et al. [IceCube Collaboration], Astrophys. J. 833, no. 1, 3 (2016) doi:10.3847/0004-637X/833/1/3 [arXiv:1607.08006 [astro-ph.HE]].

[3] M. G. Aartsen et al. [IceCube, Fermi-LAT, MAGIC, AGILE, ASAS-SN, HAWC, H.E.S.S., INTEGRAL, Kanata and Kiso and Kapteyn and Liverpool Telescope, Subaru, Swift/NuSTAR, VERITAS and VLA/17B-403 Collaborations], Science 361, no. 6398, eaat1378 (2018) doi:10.1126/science.aat1378 [arXiv:1807.08816 [astro-ph.HE]].

[4] M. G. Aartsen et al. [IceCube Collaboration], Science 361, no. 6398, 147-151 (2018) doi:10.1126/science.aat2890 [arXiv:1807.08794 [astro-ph.HE]].

[5] Huchra, John P. et al.. The Astrophysical Journal Supplement 199,1,26: 22 pages (2012) doi:10.1088/0067-0049/199/2/26 [arXiv:1108.0669 [astro-ph.CO]].

[6] M. G. Aartsen et al. [IceCube Collaboration], Astrophys. J. 835, no. 2, 151 (2017) doi:10.3847/1538-4357/835/2/151 [arXiv:1609.04981 [astro-ph.HE]].

[7] M. G. Aartsen et al. [IceCube Collaboration], Astrophys. J. 849, 67 (2017) doi:10.3847/1538-4357/aa8dfb [arXiv:1707.03416 [astro-ph.HE]].

[8] J. Braun, J. Dumm, F. De Palma, C. Finley, A. Karle and T. Montaruli, Astropart. Phys. 29, 299 (2008) doi:10.1016/j.astropartphys.2008.02.007 [arXiv:0801.1604 [astro-ph]].

[9] M. G. Aartsen et al. [IceCube Collaboration], Astrophys. J. 835, no. 2, 151 (2017) doi:10.3847/1538-4357/835/2/151 [arXiv:1609.04981 [astro-ph.HE]]. 